\title{
Chile: congestión portuaria y racionamiento eficiente en la transferencia de carga
}

\author{
Claudio A. Agostini y Eduardo H. Saavedra
}

RESUMEN

\begin{abstract}
El sistema de precios difícilmente puede eliminar la congestión en la transferencia de carga portuaria en períodos de máxima afluencia (peak), pues el uso del puerto no depende tanto de factores estacionales como sobre todo de la llegada simultánea de barcos, situación que conduce al racionamiento de su uso. En este artículo se muestra que el racionamiento eficiente debe hacerse de acuerdo con el valor de la carga transferida más que según el orden de arribo de las naves (first-come-first-served). Se evidencia que en un racionamiento eficiente se da prioridad a la carga en contenedores, luego a la fraccionada y por último a la carga en graneles. Se aplican estos resultados a la transferencia de carga en el puerto concesionado de San Antonio Terminal Internacional de Chile.
\end{abstract}

PALABRAS CLAVES

CLASIFICACIÓN JEL

AUTORES
Puertos, transporte marítimo, transporte de mercancías, tarifas portuarias, administración de puertos, eficiencia comercial, modelos matemáticos, estudios de casos, Chile

D22, D45, L23, L90

Claudio A. Agostini es profesor titular en la Escuela de Gobierno de la Universidad Adolfo Ibáñez, Chile. claudio.agostini@uai.cl

Eduardo H. Saavedra es profesor adjunto del ilades - Universidad Alberto Hurtado, Chile. saavedra@uahurtado.cl 


\section{I}

\section{Introducción}

Las transferencias de carga portuaria utilizan una infraestructura que, debido al monto y lo prolongado del tiempo de maduración de su inversión, puede caracterizarse como un insumo esencial que presenta severas restricciones en su capacidad en períodos de máxima afluencia o de alta demanda. En general, en la teoría económica se propone que las congestiones deben ser eliminadas o reducidas en forma eficiente mediante el sistema de precios. Lamentablemente, este mecanismo no puede aplicarse a los puertos, como sí puede hacerse al consumo eléctrico o de agua potable, ya que el uso de la infraestructura portuaria no depende tanto de factores estacionales, como de la llegada casi simultánea de un excesivo número de barcos. Esto plantea la necesidad de racionar el uso del puerto, generando la espera de algunos barcos.

Sin embargo, cómo llevar a cabo el racionamiento es un tema en disputa. Si bien el criterio más conocido para racionar es simplemente según el orden en que se efectúa (first-come-first-served), en la literatura económica se ha mostrado la alta ineficiencia de este criterio, dado que la disposición a pagar de los operadores racionados difiere notablemente de acuerdo con el valor de la carga transportada (Strandenes y Wolfstetter, 2005; Button, 1979), generando además mayores costos en la operación del puerto (Imai, Nagaiwa y Chan, 1997).

En concordancia con la literatura, en este artículo se muestra de manera teórica y empírica que es socialmente deseable que se asigne todo el racionamiento a las actividades que tienen menor valor agregado. Dicho valor se mide en forma abstracta por la caída en el valor de la carga transportada cuando esta es racionada. El resultado se mantiene si al modelo se incorporan compensaciones a las empresas racionadas o si la capacidad de embarque del puerto se ve afectada según con qué tipo de servicio se raciona. El criterio de racionamiento utilizado en este trabajo es por valor de la carga, distinguiendo la carga en contenedores de aquella en graneles. Ambos tipos de carga presentan marcadas diferencias en su valor, así como en el costo para el operador del puerto, en términos de tiempos de operación y uso de la infraestructura portuaria principalmente.
Este trabajo está motivado por una disputa presentada en Chile ante el Tribunal de Defensa de la Libre Competencia (TDLC) en el año 2007. La empresa Terquim S.A. acusó a San Antonio Terminal Internacional (STI) y a la Empresa Portuaria San Antonio (EPSA) de abuso de posición dominante, por dar atención a las naves en el puerto utilizando un criterio de prioridad y no de espera según el orden de arribo (first-come-first-served). Sin atender al argumento planteado por Terquim, y en consistencia con el presente trabajo, el TDLC desechó la acusación en enero de 2010, veredicto que fue ratificado por la Corte Suprema en septiembre del mismo año ${ }^{1}$, al utilizar prioridades de atención a las naves en el puerto del criterio de "first-come-first-served".

Sin embargo, más allá de esa disputa de libre competencia en lo puntual, los argumentos económicos para racionar la infraestructura portuaria en un período de tiempo en particular son aplicables a cualquier puerto, así como también lo es la metodología propuesta en este artículo.

El resto de este trabajo se organiza de la siguiente forma. En la sección II se describe el marco institucional con respecto a la propiedad público-privada de los puertos en Chile, las particularidades del puerto de San Antonio y las dos empresas que allí operan (STI y EPSA), y la principal carga a granel que transfiere la empresa STI (ácido sulfúrico). En la sección III se revisa la literatura de racionamiento en puertos y se describe la naturaleza de facilidad esencial de la infraestructura portuaria. En la sección IV se presenta un modelo económico que muestra por qué un racionamiento por valor de la carga es más eficiente que un racionamiento por orden de llegada. En la sección V se ofrecen estimaciones comparativas según se siga un criterio u otro para el puerto de San Antonio, utilizando información de la STI del año 2007. Finalmente, en la sección VI se entregan las conclusiones.

\footnotetext{
${ }^{1}$ Para mayores detalles véanse Agostini y Saavedra (2008), la sentencia 96/2010 del TDLC y el fallo de la Corte Suprema caratulado como Rol 1933/2010. Véase [en línea] www.tdlc.cl.
} 


\section{II}

\section{Marco institucional y descripción del mercado}

\section{Las asociaciones público-privadas de puertos en Chile}

Durante las décadas de 1980 y 1990, la Empresa Portuaria de Chile (EMPORCHI) operaba los 10 puertos de propiedad del Estado mediante un sistema de operación llamado multi-operador. Bajo este sistema, la empresa estatal administraba la infraestructura portuaria y varias empresas privadas realizaban las labores de carga y descarga de naves en los puertos. Una de las grandes desventajas de este sistema es que divide la carga entre varias empresas en un mismo puerto, lo que limita seriamente los incentivos para invertir en equipos de transferencia e impide el uso eficiente de la infraestructura portuaria.

Dado el fuerte crecimiento experimentado por el comercio internacional en Chile, la gestión portuaria se comenzó a transformar en un cuello de botella y a fines de los años noventa el gobierno tomó la decisión de modernizar el sector portuario estatal. Los objetivos centrales de la reforma fueron impulsar y dinamizar el proceso de inversión en infraestructura, tecnología y gestión portuaria. Para ello, se propuso reemplazar el sistema multi-operador por un sistema mono-operador, en el que una sola empresa se hace responsable de la operación y mantenimiento de un terminal portuario. De esta forma sería posible promover la competencia tanto entre puertos como en la etapa de licitación del futuro mono-operador. Es así como se planteó una reforma tendiente a dividir la EMPORCHI, incorporar la participación de privados en el desarrollo de puertos estatales a través del mecanismo de concesiones y modernizar la gestión laboral de los puertos.

La reforma aprobada en 1998 creó 10 empresas portuarias estatales autónomas, cada una dueña de un solo puerto, con el objetivo explícito de administrar, explotar, desarrollar y conservar sus respectivos puertos y terminales. Además, la ley estableció como misión de cada una de estas empresas promover la competencia entre puertos y en el interior del puerto, e incorporar la participación del sector privado para incrementar la eficiencia y la inversión. Para ello, las empresas portuarias pueden licitar la concesión a privados para la operación y la realización de inversiones en cada uno de los terminales portuarios de su propiedad. Bajo el esquema de concesión, cada empresa portuaria estatal se mantiene como dueña de la infraestructura y fiscaliza el contrato de concesión, recibiendo como ingresos una renta mínima anual por parte del concesionario junto con un porcentaje de sus ingresos.

En 1999 se llamó a licitación para concesionar los tres principales terminales portuarios en Chile: San Antonio, Valparaíso y San Vicente (Talcahuano), los que sumados representaban del orden de $50 \%$ del total de carga transferida por EMPORCHI. En la licitación se consideraron dos criterios de adjudicación: i) por un índice tarifario calculado a partir de las tarifas de muellaje a la nave, muellaje a la carga, transferencia de contenedores y transferencia de carga fraccionada, y ii) por un pago al Estado o canon anual.

A continuación, este artículo se concentra en la actividad de carga en el puerto de San Antonio, el de mayor transferencia de carga total y el segundo en carga en contenedores en Chile, de acuerdo con cifras de la Unidad de Servicios de Infraestructura de la CEPAL para el año 2011.

\section{Las empresas portuarias públicas y privadas en San Antonio}

La empresa estatal EPSA posee cuatro frentes de atraque con un total de 9 sitios y una superficie total de 495 hectáreas, de las cuales 353 corresponden a áreas marítimas y 142 a áreas terrestres. La poza de abrigo tiene una superficie de 75 hectáreas y los cuatro terminales son el Molo Sur (sitios de atraque 1, 2 y 3), el Espigón (sitios de atraque 4, 5, 6 y 7), el Terminal Norte (sitio 8 especializado en graneles sólidos), y el sitio 9, especializado en graneles líquidos.

En el proceso de licitación del puerto de San Antonio se contemplaron las concesiones del Molo Sur y el Terminal Norte. El Molo Sur, con los sitios de atraque de mayor capacidad, fue adjudicado a San Antonio Terminal Internacional (sTI), con un índice tarifario de 7,05 dólares la tonelada, un pago por adelantado (upfront) de 10.000.000 de dólares, un canon anual que dado el tonelaje movilizado en el año 2007 alcanzó a 11.050.606 de dólares y un pago adicional de 121.252.062 de dólares (pagaderos en seis cuotas anuales de igual monto en los primeros seis años de adjudicada la concesión). El Terminal Norte fue adjudicado a Puerto Panul y los cinco sitios restantes son administrados por EPSA. Así, STI es la empresa concesionaria que opera y administra el 
terminal Molo Sur, especializado en contenedores. Para estos efectos cuenta con 769 metros de muelle continuo, 12 metros de profundidad a lo largo de su frente de atraque en el momento de la concesión ${ }^{2}, 31$ hectáreas de explanadas de las cuales 25 están destinadas al acopio de contenedores y cargas a granel, 6 grúas pórtico, 9 grúas horquilla, 41 tracto-camiones para la manipulación de contenedores y carga dentro del terminal, un área para consolidación y desconsolidación de contenedores, 6.000 metros cuadrados de almacenaje techado para carga, 2.000 conexiones para carga refrigerada (reefer) disponibles, acceso de ferrocarriles hasta el costado de las naves y zonas de carguío de contenedores, y romana para el pesaje de camiones con carga a granel o contenedores.

En el contrato de concesión con sTI se establecen velocidades de transferencia y tiempos de espera que deben cumplirse, y en caso de incumplimiento el concesionario es multado. Además, hay reglas establecidas para una mejoría progresiva en el servicio que presta el concesionario a lo largo del contrato. De

${ }^{2}$ Actualmente tiene 380 metros con calado autorizado de 13,5 metros y 389 metros con calado autorizado de 11,34 metros. esta forma, se incentiva la inversión necesaria por parte del concesionario para mantener y mejorar el estándar de servicio sin la necesidad de imponer inversiones específicas o montos de inversión. Las tarifas básicas están fijadas en el contrato de concesión; sin embargo, el concesionario puede cobrar tarifas especiales por servicios adicionales que provea previa solicitud del usuario. Esto estimula al concesionario a invertir de acuerdo con la evolución tanto del progreso técnico en las operaciones portuarias como de la demanda de sus clientes de diferentes tipos, los que requieren diferentes niveles de servicio.

Debido a que el uso de contenedores ha producido una importante reducción en los costos de manejo de la carga, aumentando así el cabotaje nacional e internacional (Clark, Dollar y Micco, 2004; Blonigen y Wilson, 2008), uno de los objetivos de la licitación era precisamente que se realizaran inversiones que permitieran aumentar la capacidad y la eficiencia de la transferencia de carga en contenedores. Esta tendencia también se observa en el puerto de San Antonio, tanto en la evolución del número de atraques en cada terminal por tipo de barco (véase el gráfico 1), como en el total de carga transferida por tipo de barco (véase el gráfico 2) y en el rendimiento por tipo de carga transferida (véase el gráfico 3).

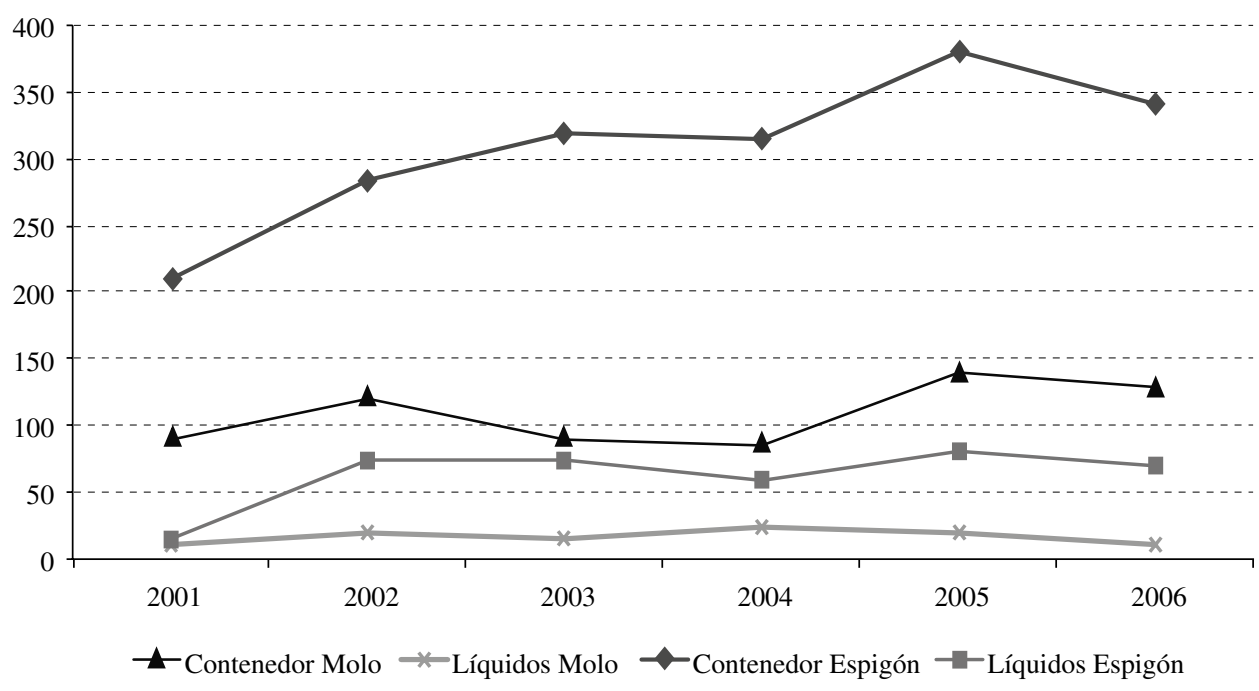

Fuente: elaboración propia sobre la base de información proveniente del San Antonio Terminal Internacional (STI), Chile. 
GRÁFICO 2 STI: toneladas transferidas por tipo de carga, 2000-2006

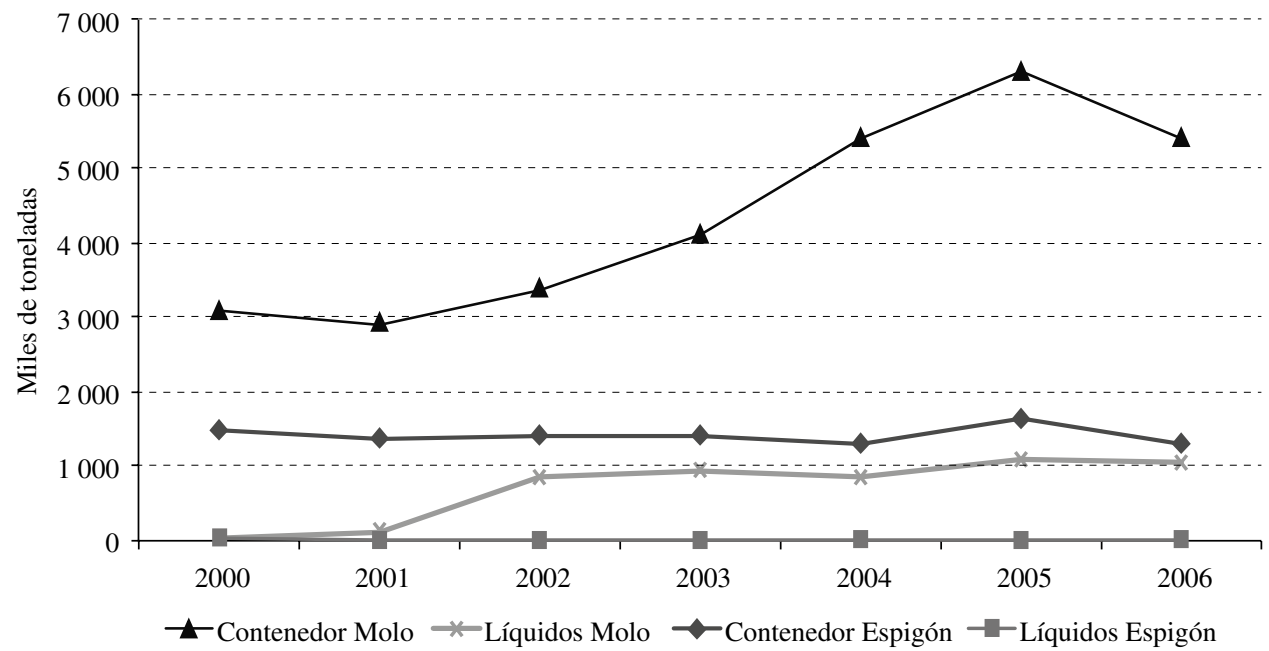

Fuente: elaboración propia sobre la base de información proveniente del San Antonio Terminal Internacional (sti), Chile.

GRÁFICO 3 STI: rendimiento por tipo de carga transferida, 2000-2006 (Toneladas/hora)

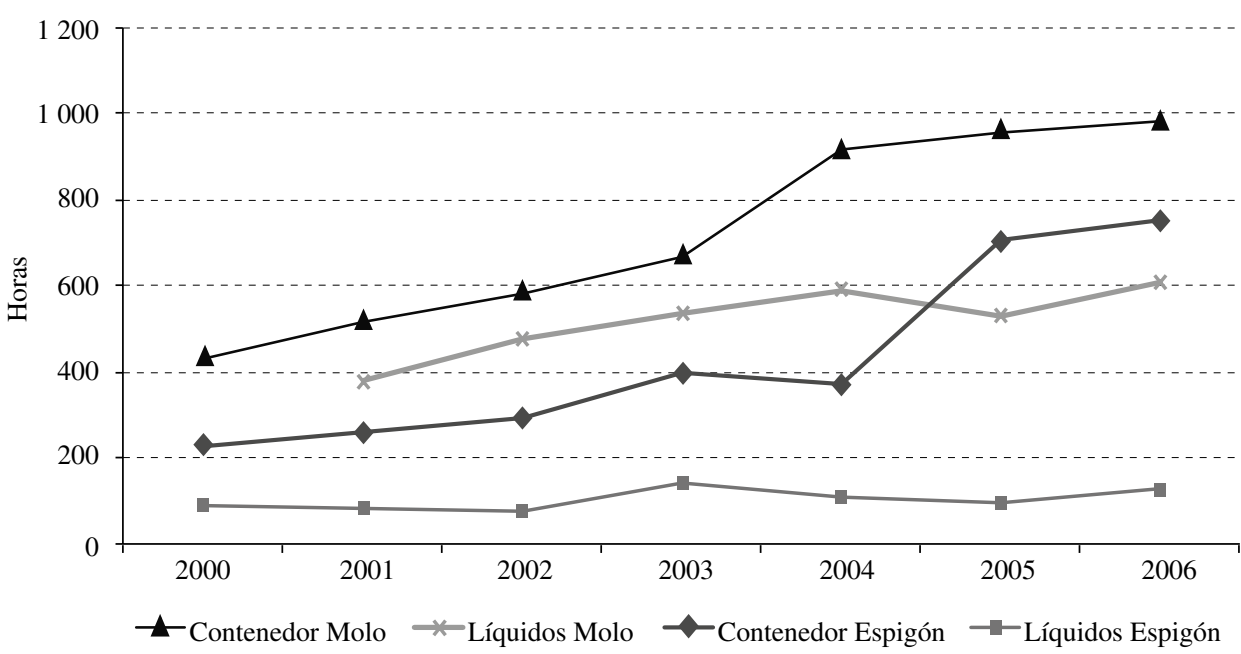

Fuente: elaboración propia sobre la base de información proveniente del San Antonio Terminal Internacional (sTi), Chile. 
La evolución que ha tenido la carga en los últimos años, reflejada en los gráficos 1, 2 y 3 , no solo muestra la tendencia a una mayor utilización de contenedores, sino también la mayor eficiencia portuaria que se obtiene con el uso del contenedor. Por ello, la especialización de frentes de atraque en carga en contenedores permite obtener ganancias de eficiencia con respecto a frentes de atraque en que se mezclan graneles y contenedores. Esto es relevante, ya que en puertos ineficientes se puede reducir en forma considerable el volumen de comercio y el impacto puede ser aún mayor en países pequeños y en desarrollo (Blonigen y Wilson, 2008).

Los sitios de atraque son un bien de uso público, lo que implica tarifas públicas no discriminatorias, atención de naves y movilización de carga. Por ello, en todos los puertos concesionados existe un reglamento interno de uso de frentes de atraque cuyo objetivo es el uso eficiente de la infraestructura portuaria y la libre elección de los usuarios. En el manual se determinan las normas y los procedimientos para las prioridades en el atraque de naves, estableciéndose que la programación de atraque se hace sobre la base de una regla de prioridad técnica objetiva. En el cuadro 1 se muestran las prioridades establecidas en los tres sitios de atraque concesionados al STI. Estas prioridades reflejan preferencias por tipos de carga con mayores rendimientos de transferencia y de servicios portuarios que operan naves regularmente.

Chile: prioridades establecidas para sitios del STI

\begin{tabular}{llll}
\hline & \multicolumn{1}{c}{ Sitio 1 } & \multicolumn{1}{c}{ Sitio 2 } & \multicolumn{1}{c}{ Sitio 3 } \\
\hline $1^{\circ}$ & $\begin{array}{l}\text { Naves de contenedores de } \\
\text { servicio regular } \\
\text { Naves de carga fraccionada de } \\
\text { servicio regular }\end{array}$ & $\begin{array}{l}\text { Naves de contenedores de } \\
\text { servicio regular } \\
\text { Naves de servicio regular que } \\
\text { embarquen más de 10 000 } \\
\text { toneladas de carga homogénea }\end{array}$ & $\begin{array}{l}\text { Naves que embarquen más } \\
\text { de } 10 \text { 000 toneladas de carga } \\
\text { homogénea }\end{array}$ \\
& Naves graneleras & Naves de carga fraccionada de & Naves de carga fraccionada de \\
$3^{\circ}$ & Servicio regular & Naves graneleras \\
$4^{\circ}$ & Otras naves & Naves graneleras & Otras naves \\
\hline $5^{\circ}$ & & Otras naves &
\end{tabular}

Fuente: San Antonio Terminal Internacional (sTI), Chile.

\section{Contrato de almacenamiento y protocolo de embarque del ácido sulfúrico}

El transporte del ácido sulfúrico desde la Corporación Nacional del Cobre de Chile (CODELCo)-El Teniente hasta el puerto de San Antonio se realiza en tres etapas secuenciales: camiones, ferrocarril y, ya en San Antonio, se almacena en estanques y se embarca. Esta última etapa es realizada por Terquim, siendo de ácido sulfúrico el 97\% de las transferencias de Terquim. Esta empresa tiene además la concesión para operar en Molo Sur, es decir, en los terminales concesionados a STI.

Si bien STI no sigue la política de reserva de sitios $\mathrm{y}$, por lo tanto, no se compromete a priorizar naves en desmedro de otras más allá de lo estipulado en su Manual de Servicios, el contrato entre sTi y codelco hace responsable a sTI de los problemas ambientales que produciría el rebalse de los terminales de Terquim por indisponibilidad de sitio de atraque. Con esta medida, en el contrato se procura minimizar los tiempos de espera a la gira de las naves que transportan ácido sulfúrico para CODELCO y, por ello, compromete al concesionario con tiempos de espera máximos para la atención de estas naves. Para tales efectos, el tiempo empieza a correr desde que el buque arriba a la estación de prácticos hasta el momento en que comienzan las maniobras para su atraque. Las multas fijadas en el contrato y vigentes al año 2007 eran de 20.000 dólares diarios (a prorrata si los tiempos son menores). Además, el costo de sacar el buque a la gira es por cuenta del STI (remolcadores, tiempo, y otros) si decide hacerlo. CODELCO a su vez paga una tarifa fija por tonelada de ácido, la que a abril de 2008 era de 1,05 dólares.

El contrato establece tres niveles de ácido sulfúrico en los estanques y el compromiso de espera máxima de parte del sTi para la atención de las naves dependiendo del volumen acumulado. Los niveles de volumen y la espera máxima son: nivel verde, consistente en menos 
de 26.000 toneladas y espera máxima de 48 horas; nivel amarillo, entre 26.000 y 33.000 toneladas y espera máxima de 24 horas; y nivel rojo, más de 33.000 toneladas y espera máxima de 6 horas.

En principio, este contrato es una solución económica eficiente, ya que es consistente con la literatura de racionamiento en puertos, tal como se evidencia en la sección III, y con la predicción teórica desarrollada mediante un modelo económico de racionamiento eficiente en la sección IV. Por una parte, este criterio de racionamiento le da ciertas garantías a coDELCo de que los estanques de ácido sulfúrico no se copen y tengan siempre capacidad para el acopio del ácido que produce su fundición. Por otra, STI tiene un costo alternativo al utilizar un sitio de atraque para un barco con contenedores, pagando por mantener un barco de ácido esperando más tiempo que el estipulado en el contrato. El efecto económico que tiene este contrato consiste precisamente en dar las señales adecuadas para racionar en forma eficiente el uso de un frente de atraque cuya capacidad es limitada.

\section{III}

\section{Literatura económica sobre racionamiento}

A continuación se revisa la literatura sobre racionamiento del uso de un bien, y en particular de la infraestructura portuaria. La sección finaliza con una breve discusión acerca de insumos esenciales, debido a las características propias del bien que se congestiona en este caso.

\section{Racionamiento y racionalidad económica}

La necesidad de racionar el uso de un bien se produce cuando es costoso modificar los precios (tiempo de espera en restaurantes), cuando el racionamiento señaliza calidad (atención médica o bienes de lujo), o cuando hay aumentos temporales de demanda y existen costos de cambio para los consumidores. Como resultado, se observan mercados en los que el exceso de demanda no conduce a un aumento de precios, sino a un racionamiento de los consumidores. Esto es cierto en mercados tan diversos como restaurantes, componentes electrónicos, semiconductores, computadores personales, metales, dióxido de titanio, polipropileno, petroquímicos, discos compactos y juguetes de niños (Mackinnon y Olewiler, 1980; Ghemawat, 1986; Basu, 1987; Carlton, 1991; Slade, 1991; Ungem-Sternberg, 1991; Haddock y McChesney, 1994; y De Graba, 1995).

A partir de esta evidencia en distintos mercados, la literatura económica se ha concentrado en tratar de explicar la existencia de racionamiento por tiempo como una situación de equilibrio, y también en determinar los mecanismos óptimos de racionamiento cuando no es posible o deseable el ajuste por la vía de aumentar los precios.

En uno de los artículos seminales en esta literatura, Barzel (1974) estableció la racionalidad económica detrás del racionamiento mediante espera por orden de llegada, al señalar que dicha espera simplemente establece un costo adicional para los consumidores de un bien. Cuando un bien está disponible en cantidades limitadas, la combinación tiempo-precio juega el mismo papel que el precio monetario cuando no hay restricción de cantidad; no obstante, se produce una pérdida de eficiencia con respecto al equilibrio sin racionamiento. En el caso en que no existen limitaciones en la disponibilidad del bien, pero hay rigidez de precios, la lógica es equivalente a la de Barzel y el tiempo de espera juega simplemente el papel de reducir el exceso de demanda hasta equilibrarlo con la oferta del bien (Alderman, 1987).

Sin embargo, incluso en el caso de que no existan rigideces de precios puede ser óptimo para una empresa racionar en vez de aumentar los precios. Bose (1996) muestra que cuando hay usuarios con diferente disposición a pagar y esta característica es desconocida por el oferente, los tiempos de espera se convierten en un mecanismo eficaz para discriminarlos, al punto que quienes esperan tienen una demanda mayor y por lo tanto una mayor disposición a pagar. Como resultado, existe un equilibrio con racionamiento en el que para el oferente es más rentable racionar consumidores que cobrar precios más altos para equilibrar el mercado.

Más allá de las distintas explicaciones teóricas que se han dado en la literatura para explicar la existencia de racionamiento como una situación de equilibrio en un mercado, lo relevante para este caso es considerar cuán óptimos son los distintos mecanismos de racionamiento. Una primera contribución proviene de Greenberger (1966), quien señaló que el sistema de prioridades óptimo depende del objetivo, ya que existe un conflicto entre 
minimizar el tiempo de espera promedio y la varianza de dichos tiempos. Es así como una regla en que se prioriza la atención de consumidores que requieren ser atendidos en menos tiempo permite minimizar la media de espera y el número de consumidores en espera, pero a costa de aumentar la varianza. Por el contrario, una regla de atención por orden de llegada permite controlar la varianza de los tiempos de espera.

En ambos criterios de racionamiento se considera que el costo de la espera es el mismo para todos los consumidores. Si ese supuesto no se cumple, surgen otras alternativas más eficientes para establecer prioridades de acuerdo con la importancia o la urgencia que tengan distintos tipos de consumidores. Es así como Pestalozzi (1964) y Likens (1976) muestran que utilizar un índice de prioridad para los aeropuertos es más eficiente que atender mediante un sistema de atención por orden de llegada. En particular, en el trabajo de Pestalozzi se evidencia que si el objetivo es minimizar el costo de demora promedio, resulta óptimo introducir prioridades por tipo de avión entre aquellos que aterrizan y los que despegan, manteniendo la regla de que los aterrizajes mantienen prioridad sobre los despegues. Es importante destacar que en todos los casos que se simulan, la regla de atención por orden de llegada nunca resulta óptima.

$\mathrm{Al}$ considerar distintas reglas para la atención de computadores, Greenberger (1966) establece que lo óptimo es atender a los usuarios de acuerdo con el costo de espera de cada uno, atendiendo primero a los que tienen un mayor costo. Este resultado es similar al que ya habían obtenido Cox y Smith (1961), quienes mostraron que cuando hay heterogeneidad en los costos de atraso en el servicio, el costo promedio por el atraso para los consumidores se minimiza atendiéndolos en orden descendente en la lista de prioridad, que se define como el costo de espera por unidad de tiempo dividido por el requerimiento de servicio esperado.

Posteriormente, Naor (1969) mostró que la regla de atención por orden de llegada con una población homogénea de consumidores genera un grado de congestión que excede el socialmente óptimo, por lo que es necesario aumentar el precio (o cobrar una tarifa adicional) a un nivel que reduzca la congestión. Este resultado fue posteriormente extendido por Balachandran y Schaefer (1979) para el caso en que hay consumidores heterogéneos.

En el trabajo de Sherman y Visscher (1982) se considera la estrategia de precios óptimos junto con mecanismos de racionamiento cuando la demanda por el servicio es estocástica. Sus resultados evidencian que un mecanismo de racionamiento basado en dar prioridad a los consumidores con mayor disposición a pagar, implica un precio óptimo igual para todos los consumidores. Por el contrario, un mecanismo de racionamiento en que se prioriza a los consumidores que valoren menos el servicio, implica precios óptimos discriminatorios en que se cobran precios mayores a los consumidores con mayor disposición a pagar.

Estos resultados son relevantes para el racionamiento en el uso de la infraestructura portuaria, ya que muestran que cuando no es posible cobrar precios diferentes a distintos tipos de consumidores y se obliga a cobrar un solo precio no discriminatorio, es óptimo racionar el exceso de demanda en orden descendente a la disposición a pagar.

\section{Racionamiento en el uso de la infraestructura portuaria}

En la gran mayoría de los trabajos en la literatura de racionamiento en puertos se concuerda con que el mecanismo de atención mediante espera por orden de arribo es ineficiente, a no ser que todos los arribos sean idénticos tanto en barcos como en carga. En efecto, Strandeness y Wolfstetter (2005) señalan que el criterio de atención por orden de llegada es altamente ineficiente, ya que no refleja los costos de espera relativos de los barcos. Asimismo, Imai, Nagaiwa y Chan (1997) concluyen que con el objetivo de lograr una elevada productividad portuaria, en un mecanismo de asignación óptima de sitios de atraque nunca se debe considerar como alternativa el mecanismo de atención por orden de llegada.

Entonces, por razones de eficiencia, en un puerto se debiera discriminar mediante peajes - tarifas adicionales - $\mathrm{u}$ otros mecanismos. Jansson y Ryden (1979) sugirieron utilizar una tarifa en dos partes. De tal forma que la tarifa se dividiría en un cargo que refleje el costo de oportunidad de utilizar la facilidad portuaria y otro cargo por tonelada que se diferenciaría sobre la base de la elasticidad de la demanda. De modo similar, Button (1979) evalúa el diseño de un sistema económico de precios en que se considere como criterio que los usuarios de un puerto paguen el costo de oportunidad social marginal de los recursos que utilizan. El resultado es que los puertos deben cobrar una tarifa en dos partes, consistente en un cobro por carga, basado en el costo de oportunidad marginal social, y en un cobro fijo por el derecho a hacer uso del puerto, basado en la frecuencia y el tiempo de ocupación de este. Bajo este sistema los usuarios regulares del puerto tienen prioridad sobre los usuarios infrecuentes, dado que el sistema de atención por orden de llegada no refleja la demanda efectiva de cada barco por servicios portuarios. 
En esta última línea de argumentación, Ghosh (2002) muestra que es óptimo otorgar prioridad a los barcos que más valoran el servicio y propone para ello un sistema de remate secuencial de sitios de atraque. A partir de esta idea y aplicando el Mecanismo de Groves o de Vickrey-Groves-Clarke, Strandenes y Wolfstetter (2005) proponen un sistema de licitación de sitios de atraque mediante un mecanismo que asegura que las ofertas en la licitación revelan el verdadero valor que tiene para cada barco atracar en el sitio y hora que se licita.

Más allá de las consideraciones teóricas y el consenso en la literatura con respecto a la ineficiencia de utilizar el sistema de atención por orden de llegada para asignar sitios de atraque, en la práctica distintos sistemas de priorización han sido crecientemente empleados en distintos puertos en todo el mundo. Por ejemplo, Imai, Nishimura y Papadimitriou (2004) argumentan que la asignación de sitios en que se toman en cuenta consideraciones de prioridad es de gran importancia para los operadores de puertos que funcionan en un contexto de competencia, sobre todo porque les da una mayor flexibilidad en sus decisiones de uso de la infraestructura.

En consecuencia, en algunos puertos se establece el tamaño del barco o el volumen de carga como criterio de prioridad. A modo de ejemplo, las autoridades portuarias en el Japón, Singapur y Noruega asignan prioridad en algunos puertos a los barcos con mayor volumen de contenedores (Imai, Nagaiwa y Chan, 1997; Imai, Nishimura y Papadimitriou, 2004; y Svendsen, 1967).

\section{Los puertos como facilidad esencial}

Conceptualmente, una facilidad esencial o infraestructura esencial se puede entender como el insumo básico para abastecer a empresas que participan en segmentos competitivos - $\mathrm{O}$ al menos en competencia imperfectade una industria, donde este insumo básico es provisto bajo condiciones monopólicas o con poder de mercado. Es importante destacar que el poder de mercado derivado de la propiedad o el control en la operación de los activos denominados esenciales no necesariamente requiere ser monopólico, basta con que el operador de estos activos pueda, sin regulación alguna, imponer un precio límite que le signifique rentas por sobre lo normal en su operación.

Lo primero que es necesario entender adecuadamente del concepto de facilidad esencial es que la industria debe tener una estructura vertical, es decir, que el mercado que atiende a los consumidores requiere necesariamente acceder a un insumo básico, pues insumos alternativos no existen o no son viables económicamente ${ }^{3}$. En el caso de un puerto, esta facilidad esencial interactúa con diversas empresas navieras que ofrecen el servicio de transporte de carga, ya sea en contenedores, granel o fraccionada. A su vez, hay usuarios indirectos del puerto, como los productores y los consumidores finales de los bienes transportados. Además, existe la participación de otros actores en la actividad de transporte de carga portuaria, como son la autoridad marítima y los prácticos navieros antes o después de la llegada o salida de una nave respecto del sitio de atraque, así como de aduanas y agencias de aduanas y del Servicio Agrícola y Ganadero antes o después del arribo.

Lo segundo que se debe entender es que no todo tipo de facilidad esencial requiere ser regulada. Es posible encontrar industrias con bajos costos hundidos y bajo poder de mercado en que se produce competencia por facilidades esenciales, donde dos o más empresas optan por construir su propia facilidad esencial, dando incluso acceso pagado - pero determinado libremente por el mercado - a sus rivales en el mercado del cliente final. Ejemplo de esto sería una industria de salud liberalizada (Robinson y Casalino, 1996), o la de las telecomunicaciones (Valetti y Cambini, 2005; Mancero y Saavedra, 2006). Alternativamente, existen industrias con mayores costos hundidos donde dos o más competidores comparten sus facilidades esenciales, incluso como un resultado de mercado ante la entrada de un nuevo competidor, como podría ser la industria de combustibles líquidos (Balmaceda y Saavedra, 2007) o la del transporte aéreo (Agostini, 2008 y 2012).

Si se ha optado por regular el acceso a la facilidad esencial, en tarifas y en calidad, aparecen dos elementos interesantes de analizar. Primero, en el momento de diseñar la subasta de la privatización o de la concesión portuaria se debe ser cauteloso en las condiciones $e x$ post que rijan la operación misma de las instalaciones (Engel, Fischer y Galetovic, 2004), debiéndose cuidar las condiciones respecto de la propiedad de las operadoras privadas, la estructura horizontal de la industria, la regulación tarifaria y la regulación de la calidad del servicio que se va a prestar. En segundo lugar, si se opta por no separar verticalmente la industria, el operador portuario puede tener intereses comerciales en el mercado naviero, por lo que podría sabotear a los rivales mediante la reducción de la calidad del

\footnotetext{
3 En rigor, la industria portuaria sí presenta sustitutos imperfectos en la facilidad esencial, como la existencia de otros puertos más alejados dentro del país o un puerto estatal en el mismo lugar. Estos sustitutos son imperfectos pues se puede acceder a ellos a un costo mayor (monetario o en tiempo de espera).
} 
servicio prestado con respecto al brindado a sus propias naves (Mandy y Sappington, 2007). Previniendo estos riesgos, la regulación en Chile fija explícitamente normas de prioridades de servicio, demora en la espera y estándares de calidad que son supervisadas por el regulador portuario correspondiente.

En suma, la concesión de la operación de la infraestructura portuaria es económicamente eficiente en tanto el proceso de licitación junto con el de regulación emulen un mercado competitivo, entregando al operador las señales correctas de tarificación, calidad de servicio y sobre todo de inversión. En consecuencia, no está en disputa si las inversiones portuarias han sido las adecuadas, pues la congestión con precios dados es algo que se va a producir de todos modos en algún momento, y es precisamente en ese momento cuando se requiere una respuesta eficiente para el manejo del racionamiento.

\section{IV}

\section{Un modelo de racionamiento eficiente en puertos}

\section{Modelo básico}

Dadas las decisiones tomadas por el Estado con anterioridad a la licitación de los puertos, en especial las restricciones de propiedad y de la estructura de la industria, así como la fijación de tarifas para los diversos servicios, el operador portuario se enfrenta a la necesidad de racionar el uso de la facilidad esencial en períodos de alta demanda del servicio. En términos simples, cualquiera sea el volumen de inversión necesario, lo óptimo y real es que habrá períodos en que la infraestructura portuaria tendrá capacidad disponible para atender a todo tipo de carga y, óptimamente, habrá otros períodos en que las instalaciones se saturarán por exceso de demanda.

A continuación se muestra que el racionamiento eficiente se logra asignando toda la actividad portuaria en períodos de alta demanda del servicio de contenedores, dejando la capacidad residual para otros servicios con menor valor agregado, como carga fraccionada o graneles, cuya atención es provista luego de finalizada la congestión. Esto es correcto en tanto no haya externalidades que afectan a la población como consecuencia de eventuales derrames a raíz del exceso de acumulación de graneles peligrosos en el puerto, como sería, por ejemplo, el caso del ácido sulfúrico en tiempos de alta acumulación. Esta asignación de la carga en favor de los contenedores es en general más eficiente por tres razones: mayores ganancias en términos de excedentes para los demandantes de servicios portuarios, mayores beneficios para el concesionario del puerto y mayor capacidad portuaria disponible para los servicios prestados en períodos de alta demanda ${ }^{4}$.

En el supuesto de que la actividad portuaria se restringe a solo dos actividades: transferencia de contenedores $(C)$ y transferencia de buques graneleros $(G)$, solo tres períodos son relevantes, el primero, donde no hay racionamiento pues la demanda total por ambas actividades es menor a la capacidad instalada $(K)$; el segundo, donde la demanda por transferencia de contenedores crece a tal punto que la demanda total supera a la capacidad instalada $\left(D_{l}>K\right)$, y el tercero, donde la demanda vuelve a su nivel normal y se atiende además a la demanda racionada en el período previo. Asimismo, los precios por estos servicios están regulados exógenamente y por lo tanto son iguales a $p^{C}$ y $p^{G}$, y los costos son respectivamente $c^{C}$ y $c^{G}$, donde $p^{C}>p^{G}>c^{G}>c^{C}$. Esto último capta el hecho de que el margen que obtiene la concesionaria por el servicio a contenedores es mayor que el margen obtenido por el servicio a graneles 5 .

\footnotetext{
${ }^{4}$ La mayor capacidad portuaria se entiende como la rapidez con que es servida la transferencia de carga de buques que transportan un mismo tonelaje. Así, la capacidad del puerto aumenta cuando un barco es servido más rápido que otro, manteniendo constante el tonelaje transferido.

5 Nuevamente esto es a condición de que no haya riesgos en la acumulación de graneles, en caso contrario se estaría frente a un $c^{G}$ mayor, incluso por sobre el precio cobrado por esta actividad.
} 
Las demandas por ambos servicios en el segundo y tercer períodos son iguales entre sí y dadas por $p^{C}=a-b \cdot Q^{C}$ y $p^{G}=a-b \cdot Q^{G}$, siendo la misma demanda para la actividad de graneles en el segundo período, y creciendo la demanda por el servicio de contenedores en el segundo período a $p^{C}=a+\alpha-b \cdot Q^{C}$. En el gráfico 4 se resume la estructura de esta industria para el segundo y tercer períodos, siendo la del tercero similar a la del primero.

El bienestar de la sociedad es igual a la suma de los excedentes de los demandantes más los beneficios de la empresa portuaria. Es importante subrayar la relevancia de incorporar en el beneficio social las rentas de la empresa portuaria, porque: i) con ello paga la inversión realizada en infraestructura, y ii) la actividad portuaria ha sido concesionada y por lo tanto las rentas esperadas de este negocio ya fueron capturadas por el Estado en el momento de licitar el puerto con el canon anual y el pago por adelantado (upfront) requerido. No obstante, como se muestra más adelante, los resultados cualitativos no cambian si solo se considera el excedente de los demandantes del servicio portuario.

Si llamamos $U_{t}^{C}$ y $U_{t}^{G}$ respectivamente a los excedentes netos de los demandantes de transferencia de contenedores y de graneles $(\mathrm{t}=1,2,3)$, el bienestar social es:

$$
W=W_{1}+W_{2}+W_{3}=\sum_{i=1}^{3}\left(U_{i}^{C}+U_{i}^{G}+\pi_{i}^{C}+\pi_{i}^{G}\right)
$$

Hay que notar que a pesar de tener la misma demanda en los tres períodos, los excedentes de los usuarios graneleros difieren en tanto se racione el servicio en el segundo período y se satisfaga — total o parcialmenteesa demanda en el tercer período. Para comprender de mejor manera cuáles son estos excedentes que componen el bienestar social, en el gráfico 5 se ilustran los cuatro excedentes o ganancias que corresponderían al segundo período. Simplemente, solo para efectos del gráfico 5, se supone que no hay restricción de capacidad.

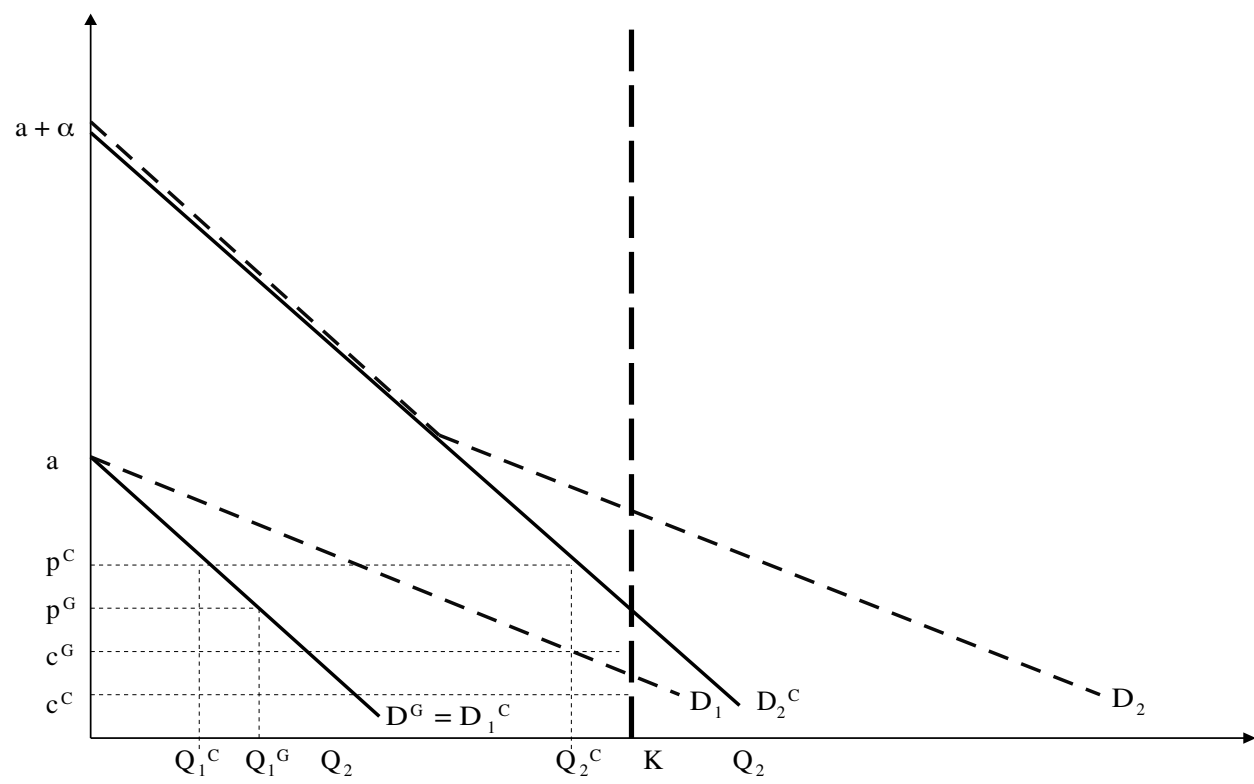

Fuente: elaboración propia sobre la base de información proveniente del San Antonio Terminal Internacional (STI), Chile. 
GRÁFICO 5

Bienestar social en segundo período sin racionamiento

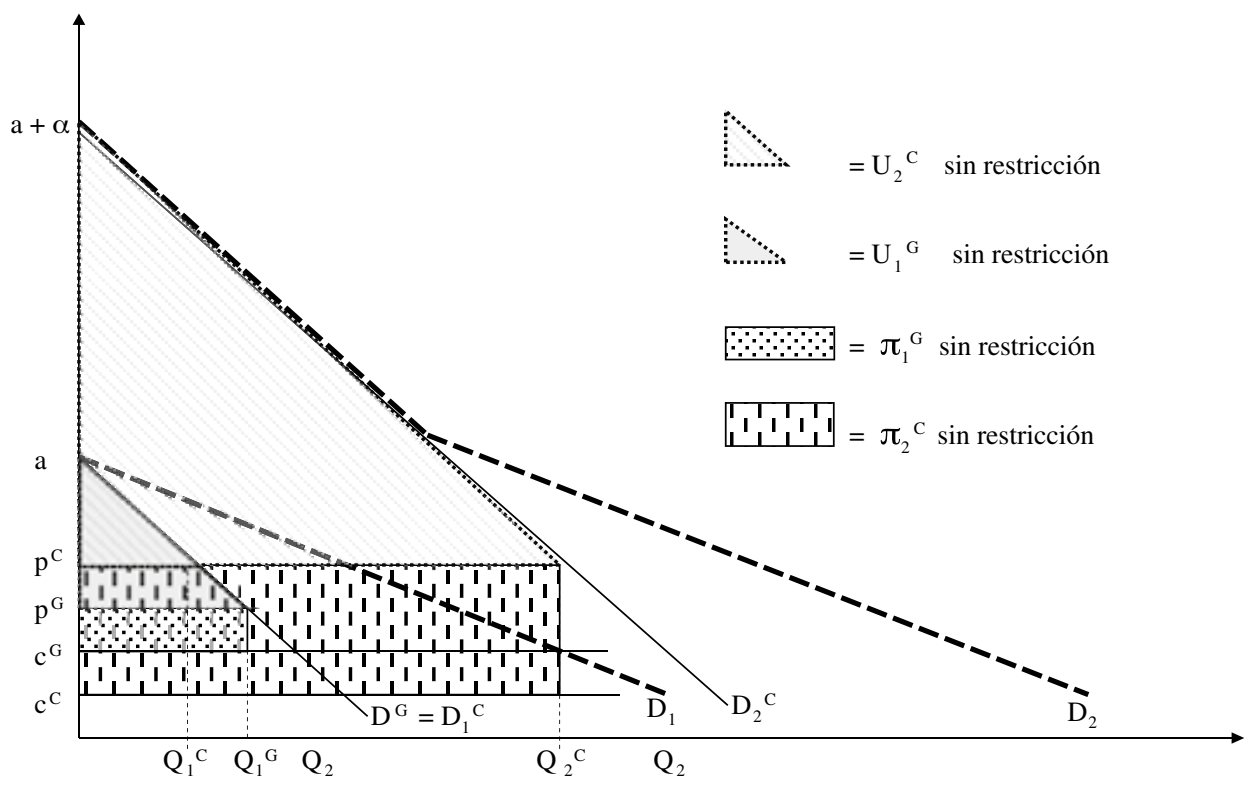

Fuente: elaboración propia sobre la base de información proveniente del San Antonio Terminal Internacional (STI), Chile.

Volviendo al caso general, con racionamiento en el segundo período se puede suponer que una proporción $\theta$ del racionamiento es asignada a la actividad de graneles, mientras que $(1-\theta)$ de ese racionamiento se asigna a la actividad de contenedores. La carga racionada es totalmente servida en el período siguiente, pero el usuario solo recibe una proporción del excedente: $\delta^{C} \mathrm{y}$ $\delta^{G}$. Dado que en la práctica la actividad de contenedores es sustancialmente más importante para la economía del país y de los propios mandantes de servicios portuarios, cabe suponer razonablemente que son estos los que más pierden con un racionamiento, lo que es capturado en el modelo, suponiendo que $0<\delta^{C}$ y $\delta^{G}<1$. Conviene aclarar que no hay descuento de los flujos entre períodos, pues se supone que el racionamiento es muy corto, por lo que $\delta^{C}$ y $\delta^{G}$ solo representan el costo de oportunidad de no ser otorgado el servicio cuando es requerido. Por último, se supone que la empresa portuaria pierde $(1-f)$ de sus ganancias al compensar a un cliente racionado, lo que cubre los costos de combustible, salarios y otros, además de eventuales compensaciones a terceros (como las que hay que pagar a CODELCo en el caso del ácido sulfúrico). En este pago no se incluyen compensaciones eventuales a las navieras racionadas, en cuyo caso solo serían traspasos de ingresos que no afectarían a la eficiencia en la asignación de recursos.
Enseguida se revisa en primer lugar el caso en que la capacidad portuaria instalada, en términos de atención de buques, es fija e independiente del parámetro $\theta$. Posteriormente se considera el caso en que esa capacidad instalada para servicios portuarios en un período determinado aumenta en tanto se asigne más racionamiento a la actividad de graneles, esto es:

$$
\frac{\partial K}{\partial \theta}>0
$$

donde $K \in[\underline{K}, \bar{K}]$.

\section{Bienestar social y racionamiento con capacidad fija}

Los excedentes de usuarios y del puerto para los tres períodos son:

$$
\begin{gathered}
W_{1}=U_{1}^{C}+U_{1}^{G}+\pi_{1}^{C}+\pi_{1}^{G} \\
=\frac{1}{2}\left(a-p^{C}\right) Q_{1}^{C}+\frac{1}{2}\left(a-p^{G}\right) Q_{1}^{G}+\left(p^{C}-c^{C}\right) Q_{1}^{C} \\
+\left(p^{G}-c^{G}\right) Q_{1}^{G}=-\frac{1}{2 b}\left[\left(a-p^{C}\right)^{2}+\left(a-p^{G}\right)^{2}\right] \\
+\frac{1}{b}\left[\left(a-c^{C}\right)\left(a-p^{C}\right)+\left(a-c^{G}\right)\left(a-p^{G}\right)\right]
\end{gathered}
$$




$$
\begin{aligned}
& W_{2}=U_{2}^{C}+U_{2}^{G}+\pi_{2}^{C}+\pi_{2}^{G} \\
& =\frac{1}{2}\left(a+\alpha-p^{C}\right) Q_{2}^{C}-(1-\theta) \frac{b}{2}\left(Q_{2}-K\right)^{2} \\
& +\frac{1}{2}\left(a-p^{G}\right) Q_{2}^{G}-\theta \frac{b}{2}\left(Q_{2}-K\right)^{2} \\
& +\left(p^{C}-c^{C}\right) Q_{2}^{C}-(1-\theta)\left(p^{C}-c^{C}\right)\left(Q_{2}-K\right) \\
& +\left(p^{G}-c^{G}\right) Q_{2}^{G}-\theta\left(p^{G}-c^{G}\right)\left(Q_{2}-K\right) \\
& =-\frac{1}{2 b}\left[\left(a+\alpha-p^{C}\right)^{2}+\left(a-p^{G}\right)^{2}\right] \\
& +\frac{1}{b}\left[\left(a+\alpha-c^{C}\right)\left(a-p^{C}\right)+\left(a-c^{G}\right)\left(a-p^{G}\right)\right] \\
& -\frac{1}{2 b}\left(2 a+\alpha-\left(p^{C}+p^{G}\right)-b K\right)^{2} \\
& -\frac{1}{b}\left[\left(p^{C}-c^{C}\right)-\theta\left[\left(p^{C}-c^{C}\right)-\left(p^{G}-c^{G}\right)\right]\right] \\
& \left(2 a+\alpha-\left(p^{C}+p^{G}\right)-b K\right) \\
& W_{3}=U_{3}^{C}+U_{3}^{G}+\pi_{3}^{C}+\pi_{3}^{G} \\
& =\frac{1}{2}\left(a-p^{C}\right) Q_{3}^{C}-\delta^{C}(1-\theta) \frac{b}{2}\left(Q_{2}-K\right)^{2} \\
& +\frac{1}{2}\left(a-p^{G}\right) Q_{3}^{G}+\delta^{G} \theta \frac{b}{2}\left(Q_{2}-K\right)^{2} \\
& +\left(p^{C}-c^{C}\right) Q_{3}^{C}+f(1-\theta)\left(p^{C}-c^{C}\right)\left(Q_{2}-K\right) \\
& +\left(p^{G}-c^{G}\right) Q_{3}^{G}+f \theta\left(p^{G}-c^{G}\right)\left(Q_{2}-K\right) \\
& =W_{1}+\frac{1}{2 b}\left(2 a+\alpha-\left(p^{C}+p^{G}\right)-b K\right)^{2} \\
& {\left[\delta^{C}+\theta\left(\delta^{G}-\delta^{C}\right)\right]} \\
& +\frac{f}{b}\left[\left(p^{C}-c^{C}\right)-\theta\left[\left(p^{C}-c^{C}\right)-\left(p^{G}-c^{G}\right)\right]\right] \\
& \left(2 a+\alpha-\left(p^{C}+p^{G}\right)-b K\right)
\end{aligned}
$$

Para efectos del análisis económico relevante, es posible separar el bienestar social en aquella parte que no depende de cómo se raciona de aquella que depende del racionamiento. Así, si se llama $W\left(a, \alpha, p^{C}, p^{G}, c^{C}, c^{G}\right) W\left(a, \alpha, p^{C}, p^{G}, c^{C}, c^{G}\right)$ a la fracción del bienestar social que no depende ni del racionamiento ni de la capacidad instalada, luego de reemplazar las ecuaciones (2), (3) y (4) en (1) se encuentra que el bienestar social total quedará expresado como:

$$
\begin{gathered}
W=W\left(a, \alpha, p^{C}, p^{G}, c^{C}, c^{G}\right) \\
+\frac{1}{2 b}\left(1-\left[\delta^{C}+\theta\left(\delta^{G}-\delta^{C}\right)\right]\right) \\
\left(2 a+\alpha-\left(p^{C}+p^{G}\right)-b K\right)^{2} \\
+\frac{1-f}{b} \theta\left[\left(p^{C}-c^{C}\right)-\left(p^{G}-c^{G}\right)\right] \\
\left(2 a+\alpha-\left(p^{C}+p^{G}\right)-b K\right)
\end{gathered}
$$

Luego, el efecto de asignar más racionamiento al servicio de graneles es siempre socialmente beneficioso, en tanto $0<\delta^{\mathrm{C}}$ y $\delta^{\mathrm{G}}<1$, pues derivando la ecuación (5) respecto de $\theta$, la derivada es positiva:

$$
\begin{aligned}
\frac{\partial W}{\partial \theta}= & \frac{1}{2 b}\left(\delta^{G}-\delta^{C}\right)\left(2 a+\alpha-\left(p^{C}+p^{G}\right)-b K\right)^{2} \\
& +\frac{1-f}{b}\left[\left(p^{C}-c^{C}\right)-\left(p^{G}-c^{G}\right)\right] \\
& \left(2 a+\alpha-\left(p^{C}+p^{G}\right)-b K\right)>0
\end{aligned}
$$

En el primer término de la expresión (6) se evidencia el cambio en el bienestar de los usuarios como consecuencia de asignar marginalmente un mayor racionamiento al servicio de menor valor agregado, lo que se refleja en que $\delta^{\mathrm{C}}<\delta^{\mathrm{G}}$. Esto es así porque los usuarios de barcos con contenedores pierden significativamente más que aquellos de barcos graneleros al atrasarse su embarque o desembarque de carga. El segundo término solo es significativo en tanto la empresa portuaria pague los costos que implica el racionamiento y se reduzcan así sus ganancias $(f<1)$, costos que son menores al servir a la actividad con mayor velocidad de transferencia de carga.

A raíz de este resultado, dado que el racionamiento es permitido debido a que los precios no se ajustan a la demanda en un momento puntual en que se entregan los servicios portuarios, es eficiente asignar todo el racionamiento a la actividad de embarque de graneles; es decir, es eficiente fijar $\theta=1$.

No obstante que este resultado es bastante general, no es aplicable del todo cuando la población se ve enfrentada a externalidades negativas propias del acopio excesivo de algunos tipos de carga, como ocurre potencialmente con el ácido sulfúrico en el caso de San Antonio o con otros materiales tóxicos en otros puertos del país. Para captar una situación como esta, el modelo puede modificarse 
para períodos de acumulación de ácido sulfúrico —el que es conocido a priori y no aleatorio-, de tal modo que el costo (social) de posponer esta carga sea creciente y convexo con la carga a granel racionada, es decir, una función $S(\theta)$, de manera que $S^{\prime}(\theta)>0$ y $S^{\prime \prime}(\theta)>0$. Bajo este supuesto, la expresión (6) se transformaría en:

$$
\left.\frac{\partial W}{\partial \theta}\right|_{S(\theta)>0}=\left.\frac{\partial W}{\partial \theta}\right|_{S(\theta)=0}-S^{\prime}(\theta) \gtreqless 0
$$

Si la carga almacenada en bodegas es suficientemente peligrosa como para que la externalidad genere costos de considerable magnitud para la sociedad, entonces este efecto dominaría y por ende, en una situación como la señalada, sería conveniente racionar a los contenedores. Cabe suponer que en general este no es el caso y por lo tanto se cumple la ecuación (6).

\section{Bienestar social y racionamiento con capacidad endógena}

En el resultado anterior con relación a la eficiencia que genera racionar el embarque de graneles o carga fraccionada respecto de la de contenedores, se asume que la capacidad portuaria está dada —es fija y exógena-, por lo que es relevante considerar si esta conclusión se mantiene en caso de que la capacidad portuaria sea endógena. Esto es sobre todo importante no solo por la robustez general de un modelo con respecto a sus supuestos, sino porque en este caso, tal como se muestra empíricamente más adelante, la evidencia señala que el tiempo destinado a la transferencia de carga de barcos graneleros es mayor que el requerido para servir a un barco con contenedores de igual tamaño.

Considerar que la capacidad portuaria es endógena a la decisión de racionamiento implica, en términos de este modelo, que la capacidad del puerto se incrementa en tanto se traspasa racionamiento desde contenedores a graneles o matemáticamente $K=K(\theta)$, con $\frac{\partial K}{\partial \theta}>0$. Se supone que $K(0)=\underline{K}$ y $K(1)=\bar{K}$; pero de todas formas, aunque se asignara todo el racionamiento a la actividad a granel, aun así persistiría el racionamiento, lo que significa que $Q_{2}>\bar{K}$. Tomando los resultados de la subsección anterior, el efecto de un aumento de $\theta$ en el bienestar social es:

$$
\begin{gathered}
\frac{\partial W}{\partial \theta}=\frac{1}{2 b}\left(\delta^{G}-\delta^{C}\right)\left(2 a+\alpha-\left(p^{C}+p^{G}\right)-b K\right)^{2} \\
+\frac{1-f}{b}\left[\left(p^{C}-c^{C}\right)-\left(p^{G}-c^{G}\right)\right]\left(2 a+\alpha-\left(p^{C}+p^{G}\right)-b K\right) \\
+\left(2 a+\alpha-\left(p^{C}+p^{G}\right)-b K\right)\left(1-\left[\delta^{C}+\theta\left(\delta^{G}-\delta^{C}\right)\right]\right) \frac{\partial K}{\partial \theta} \\
+(1-f)\left[(1-\theta)\left(p^{C}-c^{C}\right)+\theta\left(p^{G}-c^{G}\right)\right] \frac{\partial K}{\partial \theta}>0
\end{gathered}
$$

donde los últimos dos términos de (8) son nuevos -no aparecen en la expresión (6) - y captan el impacto de un menor racionamiento en tanto este se asigne a la carga/ descarga de graneles. En consecuencia, al adoptarse esta regla de priorización de la actividad portuaria tanto los efectos directos de favorecer a la carga en contenedores, como los efectos indirectos en mayor capacidad portuaria elevan el bienestar social.

Asimismo, si la repercusión en el bienestar social de racionar la carga a granel $-S^{\prime}(\theta)$ - fuera suficientemente grande, por ejemplo, como consecuencia de una mayor probabilidad de vertimiento de carga peligrosa, entonces el efecto sería contrario al predicho en la expresión (8). Se supondrá que esos casos están normados por protocolos o contratos entre el puerto y los generadores de carga, o regulados directamente por la ley o normativa ambiental, de modo que bajo esas circunstancias se aplicaría el racionamiento a los contenedores.

\section{Aplicación de criterios de prioridad en el puerto de San Antonio, Chile}

El modelo teórico que se presentó previamente, en consistencia con los resultados de la literatura económica, muestra que un mecanismo de racionamiento sobre la base de esperas en vez de precios es eficiente y aumenta el bienestar en el caso de un puerto que tiene características de facilidad esencial. La pregunta es si este modelo resulta consistente o no con el racionamiento implementado en el funcionamiento portuario en el 
mundo real, y si tales eficiencias predichas son reales o no. Con el objeto de considerar cómo funciona en la práctica un racionamiento por tipo de carga, a continuación se analiza lo ocurrido con cada uno de los barcos de transporte de líquidos que arribaron al puerto de San Antonio y utilizaron las instalaciones de STI durante el año 2007. Luego se estima la eficiencia de racionar en favor de la carga en contenedores (y carga fraccionada), por sobre la de usar el criterio según el orden de llegada para racionar la capacidad disponible cuando esta es inferior que la demanda.

En primer lugar, se procede a caracterizar cómo fue este racionamiento en el año 2007. Enseguida se muestra que el puerto es más eficiente transfiriendo carga en contenedores que en graneles o, en consistencia con el modelo de la sección IV, se muestra que $c^{C}<c^{G}$. Finalmente, se evidencia que para la sociedad es más valioso que se priorice la carga en contenedores por sobre la en granel, es decir, $p^{C}>p^{G}$. Estas estimaciones de eficiencia portuaria y bienestar se hacen por separado para el ácido sulfúrico respecto de otros líquidos.

\section{Caracterización de las esperas por racionamiento eficiente}

En el gráfico 6 se aprecia la distribución de los tiempos de espera totales para los barcos con ácido sulfúrico en el año 2007, cuantificados desde el arribo a la estación de prácticos hasta la primera espía en un sitio de atraque. De 41 barcos para el transporte de ácido sulfúrico, 15 tuvieron una espera mayor a la que hubieran tenido en caso de ser atendidos inmediatamente y sin interrupciones. Sin embargo, solo 9 de esos 15 casos son atribuibles al reglamento de prioridades (las barras negras en el gráfico 6), ya que de acuerdo con la empresa sTi en cuatro oportunidades la espera se debió a que los sitios estaban ocupados por barcos que arribaron primero, tal como operaría un puerto si siguiera el criterio según el orden de llegada (barras con tramas en el gráfico 6), y en dos casos CODELCo solicitó sacar el barco a la gira por falta de ácido sulfúrico para cargar (barras grises en el gráfico 4). El efecto de aplicar racionamiento por tipo de carga, enviando barcos graneleros a la gira, fue que ello aconteció en un $22 \%$ de los embarques de ácido sulfúrico en la sTi en 2007.

El caso de otros líquidos distintos del ácido sulfúrico no es muy diferente. En el gráfico 7 se ilustra la distribución de los tiempos de espera totales en el año 2007 para estos barcos. Se observa que ese año arribaron al puerto 22 barcos para el transporte de otros líquidos, de los cuales solo 5 tuvieron que esperar a raíz del reglamento de prioridades del sTi (barras de color negro en el gráfico 7), es decir, un 22,7\% de los barcos. Además, dos de ellos tuvieron que esperar porque los sitios se encontraban en uso por naves que arribaron con anterioridad (barras con tramas en el gráfico 7).

En resumen, el análisis de la información de esperas en el sTi durante 2007 permite concluir que un $20 \%$ de

GRÁFICO 6

STI: tiempos y causas de espera para barcos con ácido sulfúrico

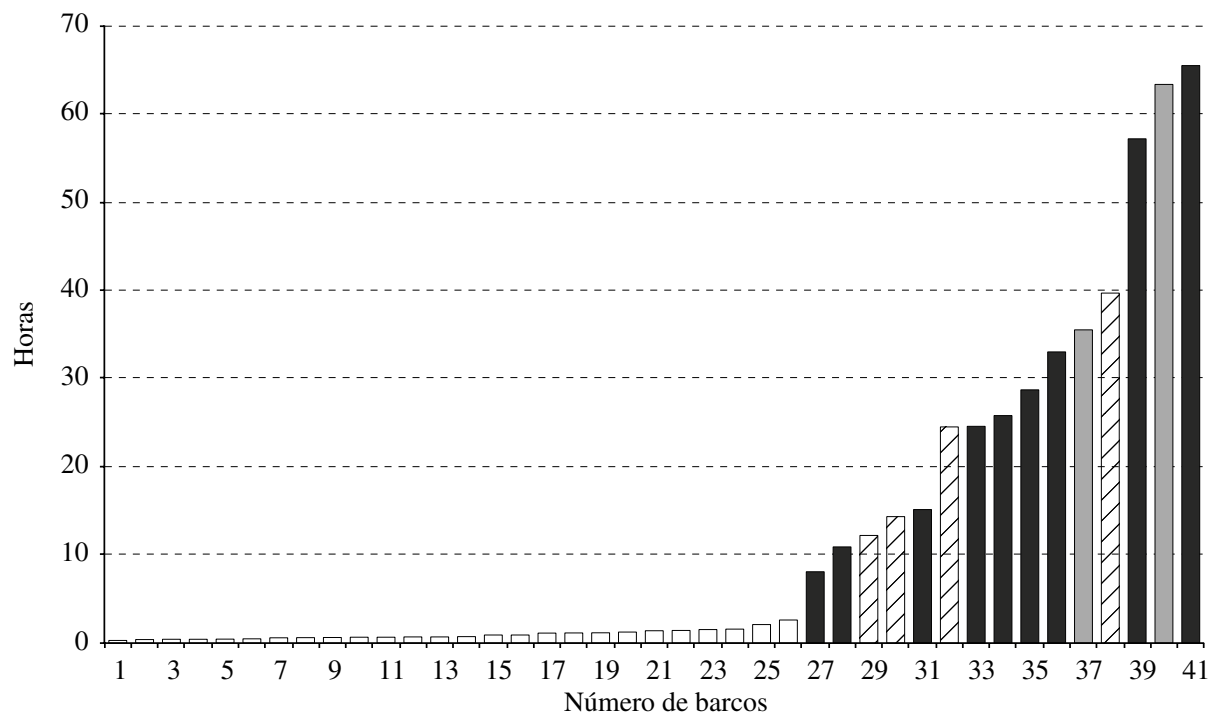

Fuente: elaboración propia sobre la base de información proveniente del San Antonio Terminal Internacional (STI), Chile. 
GRÁFICO 7

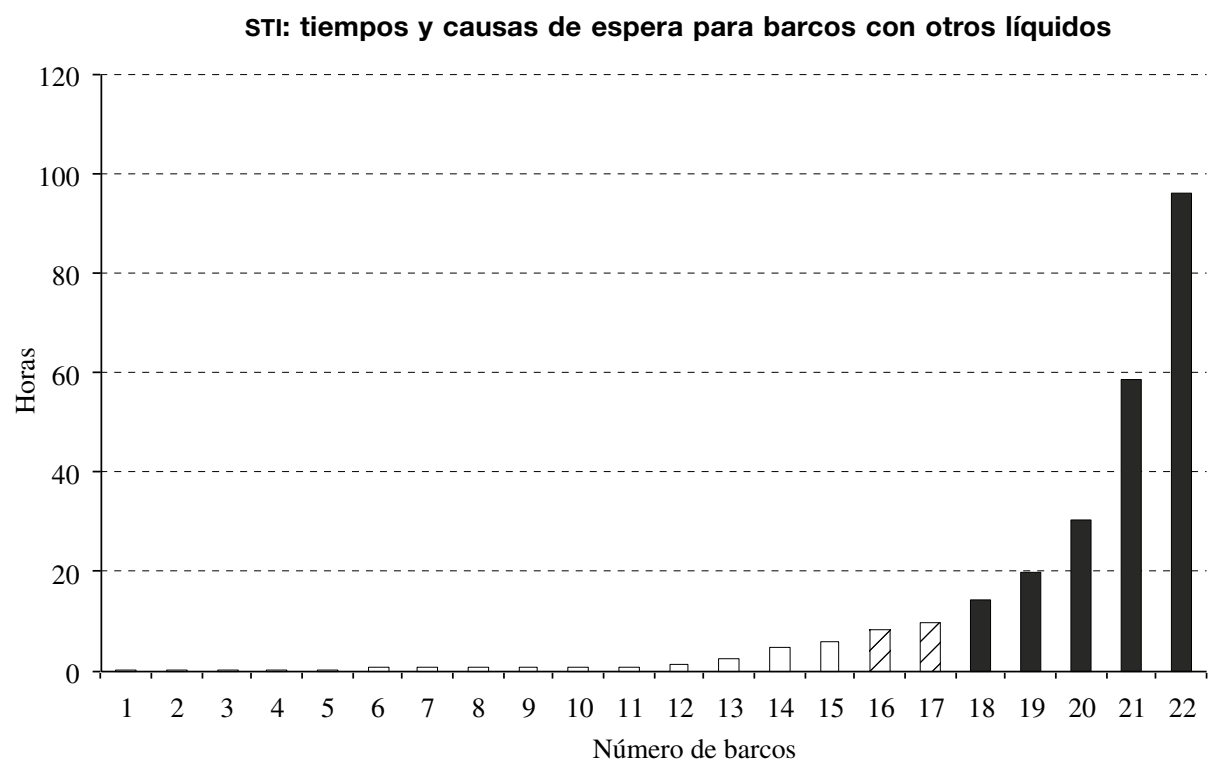

Fuente: elaboración propia sobre la base de información proveniente del San Antonio Terminal Internacional (STI), Chile.

las naves que transportaban líquidos fueron afectadas por una espera mayor a la que tendrían bajo un sistema según orden de arribo.

\section{Costo de oportunidad del racionamiento eficiente}

Más allá del porcentaje de veces que ocurre o el porcentaje de naves afectadas, es mucho más relevante conocer cuál es el costo de oportunidad de la política de racionamiento eficiente o, en otras palabras, cuál es el valor estimado de la carga en contenedores y fraccionada que tuvo prioridad sobre el ácido sulfúrico u otros líquidos. Esta información permite cuantificar de alguna forma cuánto gana la sociedad al seguir la regla de prioridades descrita, en lugar de adoptar un racionamiento por orden de llegada. Ciertamente, no se trata del bienestar social, ya que no se está estimando la demanda por cada producto, sino que se establece cuánto vale la carga a los precios de equilibrio de mercado ${ }^{6}$.

\footnotetext{
${ }^{6}$ Es importante, además, tener presente que el costo de oportunidad social de racionar la carga de ácido sulfúrico podría verse incrementado por el impacto que esta medida generaría en la producción minera que lo demanda como insumo productivo. Se supone que por tratarse de retrasos que al menos en 2007 no alcanzaron a los tres días, tal impacto no detendría los procesos productivos en la minería (en 2007, estas demoras fueron en promedio de poco menos de 30 horas).
}

\section{- Estimaciones ideales}

La carga de graneles $(G)$ tiene un valor bruto en una cierta fecha $t$ igual a $v_{t}^{G}=p_{t}^{G} \cdot Q_{t}^{G}$. Idealmente, esta información se podría obtener en forma más precisa a partir del valor $F O B$ de la carga transportada ${ }^{7}$. En tal caso el valor bruto del embarque de graneles para dicho barco sería simplemente igual a $v_{t}^{G}=F O B_{t}^{G}$. De manera similar, la carga por contenedores y la carga fraccionada (C) tienen un valor por cada barco transportado, que está dado por igual producto de precios y cantidades. Como en estos barcos la carga es heterogénea, la mejor forma de aproximarse al valor bruto de la carga transportada en cada barco $i$ en cada momento del tiempo $v_{j t}^{G}$ es simplemente tomando su valor $F O B$. Luego, si se raciona a un barco de granel para que dé paso a $J$ barcos con contenedores, con carga fraccionada o con ambos, el valor bruto para la sociedad de priorizar a los barcos que transportan este tipo de carga es $\sum_{j=1}^{J} v_{j t}^{C}=\sum_{j=1}^{J} F O B_{j t}^{C}$.

Como los costos de atender a cualquier tipo de barco son fundamentalmente fijos, pues el mayor costo es el de oportunidad de la infraestructura hundida (inversión en muelles, grúas, colector de carga (manifold), entre otros), no basta con conocer el espacio ocupado por cada barco

\footnotetext{
7 El valor FOB es el valor de la mercancía puesta a bordo del barco en el país de procedencia, excluyendo seguro y flete.
} 
en el puerto, sino además establecer el tiempo que demora cargar o descargar ese barco, según corresponda (hrs). En consecuencia, la comparación correcta es entre el valor de la carga de un barco racionado y el valor de la carga de los barcos a los que se les dio prioridad en su lugar:

$$
\frac{F O B_{t}^{G}}{h r s_{t}} \gtreqless \sum_{j=1}^{J} \frac{F O B_{j t}^{C}}{h r s_{j t}}\left(\frac{Q_{j t}^{C}}{\sum_{j=1}^{J} Q_{j t}^{C}}\right)
$$

es decir, se pondera cada operación media por los tonelajes relativos de transferencia de carga de ese barco.

No obstante, es del mayor interés conocer cuánto fue esta diferencia en un año en particular, para lo que bastaría con calcular el promedio ponderado de estas toneladas por hora para todo un año determinado:

$$
\begin{gathered}
\sum_{t=1}^{T} \frac{F O B_{t}^{G}}{h r s_{t}}\left(\frac{Q_{t}^{G}}{\sum_{t=1}^{T} Q_{t}^{G}}\right) \\
\gtreqless \sum_{t=1}^{T}\left[\sum_{j=1}^{J} \frac{F O B_{j t}^{C}}{h r s_{j t}}\left(\frac{Q_{j t}^{C}}{\sum_{j=1}^{J} Q_{j t}^{C}}\right)\right]\left(\frac{\sum_{j=1}^{J} Q_{j t}^{C}}{\sum_{t=1}^{T}\left[\sum_{j=1}^{J} Q_{j t}^{C}\right]}\right)
\end{gathered}
$$

en este caso $T$ se refiere a todos los barcos graneleros racionados en un año en particular y donde, tal como se aprecia en la fórmula, se deben ponderar las transferencias medias para cada caso.
- $\quad$ Priorización y eficiencia: ácido sulfúrico

Lamentablemente, no se encuentra información pública de los valores FOB de las transferencias realizadas, lo que permitiría hacer el cálculo con exactitud, por lo que se utilizan variables de mercado o agregadas como una aproximación a los verdaderos valores; esto es, la carga valorada a precios de mercado: $p_{t}^{a s} \cdot Q_{t}^{a s}$. Para estos efectos, se cuenta con información sobre el tonelaje de cada barco de ácido sulfúrico racionado en el año 2007 $\left(Q_{t}^{a s}\right)$, y se usa el precio promedio de este líquido pagado por los importadores chilenos, cuyo último valor CIF disponible al año 2008 era el precio promedio del año 2006: $p_{t}^{a s}=57,1$ dólares la tonelada (COCHILCO, 2007). El resto de la información relevante para los embarques de ácido sulfúrico en el año 2007 fue provisto por el STI y las variables de importancia para este cálculo se presentan en el cuadro 2.

Los datos permiten apreciar que un barco racionado demora poco menos de 30 horas en ser atendido desde que llega al puerto. Dicha cifra media es 2,5 veces mayor que la demora media en comenzar a embarcar ácido en los 41 barcos de este tipo que arribaron al puerto en el año 2007, tiempo que fue de 11,76 horas. Asimismo, la carga embarcada de ácido sulfúrico en el STI ese año fue de 573,5 toneladas por hora en cada barco racionado (promedio ponderado por los embarques de cada barco). En resumen, en el año 2007 el valor promedio de los embarques de ácido sulfúrico que transportaban estos barcos racionados por el puerto ascendió a 32.748 dólares por tonelada/hora de embarque.

\begin{tabular}{|c|c|c|c|c|c|}
\hline Arribo & Horas de espera & $\begin{array}{c}\text { Horas de carga } \\
\qquad h r s_{t}\end{array}$ & $\begin{array}{c}\text { Tonelaje } \\
Q_{t}^{a s}\end{array}$ & Toneladas/hora ${ }^{\mathrm{a}}$ & $\begin{array}{c}\text { Valor } \\
\text { (por ton/hora })^{\mathrm{b}} \\
(\text { en miles de dólares })\end{array}$ \\
\hline 2 enero & 57,15 & 44,15 & 25020 & 566,70 & 32359 \\
\hline 14 enero & 28,70 & 48,05 & 25023 & 520,77 & 29736 \\
\hline 19 febrero & 25,78 & 20,73 & 11766 & 567,49 & 32404 \\
\hline 10 marzo & 65,45 & 47,50 & 26018 & 547,75 & 31276 \\
\hline 21 marzo & 15,13 & 44,75 & 26025 & 581,56 & 33207 \\
\hline 4 mayo & 33,00 & 39,60 & 26018 & 657,02 & 37516 \\
\hline 16 agosto & 8,08 & 22,95 & 11925 & 520,36 & 29713 \\
\hline 12 octubre & 24,50 & 43,55 & 26019 & 597,45 & 34114 \\
\hline 17 diciembre & 10,90 & 26,63 & 15030 & 564,33 & 32223 \\
\hline Promedio & 29,86 & 37,54 & 21427 & 573,52 & 32748 \\
\hline
\end{tabular}

CUADRO 2

STI: barcos con ácido sulfúrico racionados en el año 2007

Fuente: elaboración propia sobre la base de información proveniente del San Antonio Terminal Internacional (STI), Chile.

a El promedio de la transferencia de los barcos racionados es ponderado por la carga de cada uno de ellos (el promedio simple entrega una carga promedio de 569,27 tons/hora).

b El promedio ponderado por carga del valor final de la carga racionada equivale a la expresión del lado izquierdo de la ecuación (10), mientras que su promedio simple entregaría un valor de 32.505 dólares por ton/hora. 
En cuanto a los barcos a los que se les dio prioridad, en desmedro de los que hemos llamado racionados, dado que se desconoce el valor FOB de cada carga y los tonelajes y precios de ellas, se utiliza el precio promedio implícito de las exportaciones e importaciones chilenas a partir de información del Banco Central de Chile (valor FOB de importaciones y exportaciones en el año 2006) y de la Cámara Marítima de Chile (cantidades totales importadas y exportadas en dicho año). Sobre la base de estos datos, se estima el precio promedio para el año 2006 de $p^{c o}=1.251$ dólares la tonelada ${ }^{8}$.

En el cuadro 3 se entrega información general acerca de cuántos barcos se priorizaron, sus toneladas transferidas, sus tiempos de uso del puerto en estas tareas, y en las dos columnas finales, los cálculos de tonelada por hora y el valor de dichas transferencias (de acuerdo con la expresión (10)).

Las principales conclusiones derivadas del análisis de datos respecto del uso eficiente del puerto son las siguientes:

i) $\mathrm{Al}$ comparar la eficiencia del puerto en la atención del embarque de ácido en comparación con la que atañe a la carga priorizada, sobre todo al transporte en contenedores, se encuentran resultados

\footnotetext{
${ }^{8}$ Este precio representa un límite inferior al verdadero precio promedio para ese año pues en esta variable proxy se incorpora el precio ponderado de la carga a granel, que es menor que el de la carga en contenedores y fraccionada.
}

consistentes con aquellos predichos por el modelo teórico en cuanto a la mayor eficiencia del puerto en la transferencia de carga por contenedores y fraccionada que al embarcar ácido sulfúrico. Las toneladas a transferir en los barcos priorizados por cada hora de uso de la infraestructura portuaria fueron 773,35 en promedio (ponderado) en el año 2007 , mientras que igual transferencia promedio por hora para barcos con ácido sulfúrico alcanzó a 573,62 toneladas por hora. Se puede concluir que el puerto es más eficiente transfiriendo tonelaje de carga por contenedores y fraccionada que embarcando ácido, pues este último representa solo un $74,16 \%$ de efectividad respecto de la actividad priorizada (o, dicho de otro modo, el puerto es más eficiente en promedio en un $34,84 \%$ al transferir carga en los barcos priorizados).

ii) Al mirar la eficiencia de las dos actividades comparadas desde el punto de vista de cómo la sociedad valora su uso, lo que se efectúa mediante algunos supuestos razonables acerca de los precios de los bienes transportados, los datos dejan ver que la transferencia de carga de contenedores y fraccionada tiene un valor 29,5 veces superior al de la actividad de embarque de ácido sulfúrico. En efecto, el valor de la carga de contenedores y fraccionada priorizada por el STI en el año 2007 alcanzó en promedio a 967.409 dólares por hora, mientras que el valor de la carga de ácido sulfúrico

CUADRO 3

STI: barcos priorizados por sobre aquellos con ácido sulfúrico en el año 2007

\begin{tabular}{|c|c|c|c|c|c|}
\hline Arribo & $\begin{array}{c}\text { Número de barcos } \\
J\end{array}$ & $\begin{array}{l}\text { Horas de carga } \\
\sum_{j=1}^{J} h r s_{j t}\end{array}$ & $\begin{array}{c}\text { Tonelaje } \\
\sum_{j=1}^{J} Q_{j t}^{c o}\end{array}$ & $\begin{array}{l}\text { Toneladas/hora } \\
(\text { ponderado })^{\mathrm{a}}\end{array}$ & $\begin{array}{c}\text { Valor } \\
\text { (ton/hora) } \\
\text { (en dólares) }^{\text {en }}\end{array}$ \\
\hline 2 enero & 3 & 41,98 & 20131 & 568,96 & 711732 \\
\hline 14 enero & 3 & 75,42 & 41515 & 684,96 & 856832 \\
\hline 19 febrero & 2 & 33,47 & 28559 & 875,24 & 1094857 \\
\hline 10 marzo & 6 & 96,00 & 84606 & 956,45 & 1196443 \\
\hline 21 marzo & 2 & 53,42 & 24086 & 534,39 & 668481 \\
\hline 4 mayo & 2 & 43,95 & 41964 & 1063,72 & 1330628 \\
\hline 16 agosto & 2 & 44,38 & 28664 & 824,46 & 1031333 \\
\hline 12 octubre & 3 & 179,92 & 76249 & 556,84 & 696569 \\
\hline 17 diciembre & 1 & 20,92 & 3234 & 154,61 & 193410 \\
\hline Promedio & 2,67 & 65,49 & 38779 & 773,35 & 967409 \\
\hline
\end{tabular}

Fuente: elaboración propia sobre la base de información proveniente del San Antonio Terminal Internacional (STI), Chile.

${ }^{a}$ La carga priorizada a cada barco racionado es ponderada de acuerdo con la ecuación (10) como $\sum_{j=1}^{J} \frac{Q_{j t}^{c o}}{h r s_{j t}}\left(\frac{Q_{j t}^{c o}}{\sum_{j=1}^{J} Q_{j t}^{c o}}\right)$. El promedio final de la transferencia de los barcos priorizados es a su vez ponderado por la transferencia antes calculada (el promedio simple entrega una carga promedio de 691,07 tons/hora).

b El promedio ponderado por carga del valor final de la carga priorizada equivale a la expresión del lado derecho de la ecuación (10), mientras que su promedio simple entregaría un valor de 864.476 dólares por ton/hora. 
racionada por STI en 2007 alcanzó en promedio a 32.748 dólares por hora. En consecuencia, en virtud de la diferencia en órdenes de magnitud resultante, no cabe duda alguna de la conveniencia social de priorizar el uso de la infraestructura portuaria en favor de la transferencia de carga en contenedores.

\section{- Priorización y eficiencia: otros líquidos}

Debido a la gran heterogeneidad de la carga transferida como otros líquidos no es posible conocer el precio medio de estos productos para el año 2007. Sin embargo, sí es posible mostrar que en términos de eficiencia de tonelaje por hora de uso de la infraestructura portuaria es correcta una regla que otorgue prioridad a la transferencia en contenedores y fraccionada por sobre la de otros líquidos. De acuerdo con la información relevante para transferencias de otros líquidos para el año 2007, provista por el sTi y mostrada en el cuadro 4 , se observa que los barcos racionados debieron esperar casi 44 horas en promedio, cifra que representa 3,8 veces la espera media de un barco de estas características, racionado o no, para este puerto en 2007. En el mismo cuadro se verifica que la velocidad de transferencia de carga en las naves racionadas fue de 135,4 toneladas por hora (promedio ponderado por los embarques de cada barco).

En cuanto a los barcos a los que se les dio prioridad, en el cuadro 5 se evidencia que la regla de priorización establecida por el sTi fue eficiente. En efecto, la carga priorizada con respecto a la carga de otros líquidos tuvo un uso de 821,33 toneladas por hora en promedio, lo que significa que el puerto es $500 \%$ más eficiente moviendo tonelaje de carga por contenedores y fraccionada que transfiriendo otros líquidos.

CUADRO 4

STI: barcos con otros líquidos racionados en el año 2007

\begin{tabular}{|c|c|c|c|c|}
\hline Arribo & Horas de espera & $\begin{array}{c}\text { Horas de carga } \\
\text { hrs }_{t}\end{array}$ & $\begin{array}{c}\text { Tonelaje } \\
Q_{t}^{\text {otros }}\end{array}$ & Toneladas/hora ${ }^{\mathrm{a}}$ \\
\hline 10 marzo & 58,75 & 8,72 & 786 & 90,17 \\
\hline 10 marzo & 96,25 & 17,25 & 1420 & 82,32 \\
\hline 30 junio & 19,97 & 16,75 & 3122 & 186,39 \\
\hline 25 noviembre & 14,42 & 11,00 & 948 & 86,18 \\
\hline 28 diciembre & 30,50 & 12,25 & 1649 & 134,61 \\
\hline Promedio & 43,98 & 13,19 & 1585 & 135,44 \\
\hline
\end{tabular}

Fuente: elaboración propia sobre la base de información proveniente del San Antonio Terminal Internacional (sTI), Chile.

a El promedio de la transferencia de los barcos racionados es ponderado por la carga de cada uno de ellos (el promedio simple entrega una carga promedio de 115,93 tons/hora).

CUADRO 5

STI: barcos con otros líquidos priorizados en el año 2007

\begin{tabular}{|c|c|c|c|c|c|}
\hline Arribo & $\begin{array}{c}\text { Número de barcos } \\
J\end{array}$ & $\begin{array}{l}\text { Horas de carga } \\
\sum_{j=1}^{J} h r s_{j t}\end{array}$ & $\begin{array}{l}\text { Tonelaje } \\
\sum_{j=1}^{J} Q_{j t}^{c o}\end{array}$ & $\begin{array}{l}\text { Toneladas/hora }{ }^{\mathrm{a}} \\
\text { (ponderado) }\end{array}$ & $\begin{array}{c}\text { Valor }^{\mathrm{b}} \\
\text { (ton/hora) }\end{array}$ \\
\hline 10 marzo & 2 & 19,08 & 13821 & 733,93 & 918086 \\
\hline 10 marzo & 5 & 71,42 & 57368 & 893,76 & 1118027 \\
\hline 30 junio & 1 & 17,28 & 17257 & 998,46 & 1249002 \\
\hline 25 noviembre & 1 & 14,70 & 2931 & 199,42 & 249459 \\
\hline 28 diciembre & 1 & 30,60 & 18043 & 589,64 & 737596 \\
\hline Promedio & 2,67 & 30,62 & 21884 & 821,33 & 1027428 \\
\hline
\end{tabular}

Fuente: elaboración propia sobre la base de información proveniente del San Antonio Terminal Internacional (STI), Chile.

${ }^{\text {a }}$ La carga priorizada a cada barco racionado es ponderada de acuerdo con (10) como $\sum_{j=1}^{J} \frac{Q_{j t}^{c o}}{h r s_{j t}}\left(\frac{Q_{j t}^{c o}}{\sum_{j=1}^{J} Q_{j t}^{c o}}\right)$. El promedio final de la transferencia de los barcos priorizados es a su vez ponderado por la transferencia antes calculada (el promedio simple entrega una carga prometdio de 683,04 tons/hora).

b El promedio ponderado por carga del valor total de la carga priorizada equivale a la expresión del lado derecho de la ecuación (10), mientras que su promedio simple entregaría un valor de 854,434 dólares por ton/hora. 


\section{VI}

\section{Conclusiones}

En la literatura económica en que se analiza la congestión en puertos se establece consistentemente que el mecanismo según el orden de llegada es ineficiente, con la excepción del caso en que todos los arribos sean idénticos. En efecto, desde un punto de vista de asignación de recursos es eficiente utilizar mecanismos de discriminación que permitan racionar en forma óptima un recurso escaso. En la aplicación presentada en este trabajo, se aprecia que en el STI, el puerto concesionado de San Antonio en Chile, al igual que en muchos otros puertos en el mundo, se utiliza un reglamento de prioridades que permite utilizar en forma eficiente los sitios de atraque, priorizando la carga en contenedores y la fraccionada por sobre la carga de graneles.

En este trabajo se emplea un modelo teórico simple que capta los hechos estilizados relevantes del uso de la infraestructura portuaria. Este modelo permite sacar conclusiones respecto de los beneficios de aplicar una regla de prioridad en la atención portuaria diferente de la regla alternativa de atención por orden de llegada. Es así como sus resultados permiten ver que, dada la inflexibilidad del sistema de precios en las concesiones portuarias, es necesario disponer de un criterio para asignar el racionamiento de manera eficiente. Tal criterio pone de manifiesto que es socialmente deseable, tanto para las empresas usuarias del puerto como para la empresa concesionaria, que se asigne todo el racionamiento a las actividades que tienen menor valor agregado. Este menor valor agregado ha sido medido en forma abstracta por la caída en el valor de la carga transportada cuando esta es racionada. Este resultado se mantiene si además se incorporan compensaciones a las empresas racionadas o se supone que la capacidad de transferencia del puerto se ve afectada según qué tipo de servicio se raciona.

La evidencia empírica de los sitios concesionados al STI es consistente con los resultados teóricos. Las estimaciones de la eficiencia del sTI en la transferencia de ácido sulfúrico y otros líquidos, respecto de contenedores y carga fraccionada, permiten concluir que el puerto es definitivamente más eficiente al mover tonelaje de carga por contenedores y fraccionado por hora de uso de la capacidad del puerto. Las estimaciones del presente trabajo evidencian que la eficiencia en la operación de los barcos con contenedores y carga fraccionada alcanza a un $34,84 \%$ por sobre la carga de ácido sulfúrico que desplaza, según el manual de prioridades de este puerto.

Más aún, esta mayor eficiencia, medida como el rendimiento en el uso de las facilidades portuarias, alcanza al 500\% en comparación con la de los barcos que cargan otros líquidos y a los cuales desplazaron según este mismo manual de prioridades. Similar conclusión se encuentra cuando se mide el valor de la carga transportada, siendo para el caso del ácido sulfúrico 26,6 veces menor que el valor de la carga que le desplazó.

Estos resultados permiten inferir que el criterio de racionamiento por valor de la carga transportada es perfectamente consistente con la eficiencia económica, medida como el uso óptimo de la infraestructura portuaria. Más aún, resulta evidente que el impacto en los barcos que transportan líquidos es menor, pues solo el $22 \%$ de estos tuvieron esperas superiores a las que ocurrirían en un ineficiente sistema según el orden de llegada.

\section{Bibliografía}

Agostini, C. (2012), "El mercado de transporte aéreo: Lecciones de política de una revisión de la literatura", Journal of Transport Literature, vol. 6, $\mathrm{N}^{\circ} 3$ São José dos Campos, Brazilian Transport Planning Society.

(2008), "La organización industrial del transporte aéreo en Chile”, Revista de Análisis Económico, vol. 23, № 1, ILADES/ Georgetown University.

Agostini, C. y E. Saavedra (2008), "Racionamiento eficiente en una facilidad esencial: el caso del Puerto de San Antonio", [en línea] http://www.tdlc.cl/DocumentosMultiples/Informe_Claudio_ Agostini_y_Eduardo\%20Saavedra_C_136_07.pdf.

Alderman, H. (1987), "Allocation of goods through non-price mechanisms: evidence on distribution by willingness to wait", Journal of Development Economics, vol. 25, № 1, Amsterdam, Elsevier.
Balachandran, K. y E. Schaefer (1979), "Class dominance characteristics at a service facility", Econometrica, vol. 47, $\mathrm{N}^{\circ} 2$, Nueva York, The Econometric Society.

Balmaceda, F. y E. Saavedra (2007), "Vertical integration and shared facilities in unregulated industries", Journal of Industrial Economics (Notes \& Comments), vol. 55, $\mathrm{N}^{\circ} 4$, Wiley, diciembre.

Barzel, Y. (1974), "A theory of rationing by waiting", Journal of Law and Economics, vol. 17, $\mathrm{N}^{\circ} 1$, Chicago, The University of Chicago Press.

Basu, K. (1987), "Monopoly, quality uncertainty and 'status' goods", International Journal of Industrial Organization, vol. $5, \mathrm{~N}^{\circ} 4$, Amsterdam, Elsevier.

Blonigen, B. y W. Wilson (2008), "Port efficiency and trade flows", Review of International Economics, vol. 16, $\mathrm{N}^{\circ} 1$, Wiley Blackwell. 
Bose, P. (1996), "Adverse selection, waiting lists and restaurant rationing", International Journal of Industrial Organization, vol. $15, \mathrm{~N}^{\circ} 3$, Amsterdam, Elsevier.

Button, K.J. (1979), "The economics of port pricing", Journal of Maritime Policy and Management, vol. 6, $\mathrm{N}^{\circ} 3$, Taylor \& Francis.

Carlton, D. (1991), "The theory of allocation and its implications for marketing and industrial structure: why rationing is efficient", Journal of Law and Economics, vol. 34, $\mathrm{N}^{\circ}$ 2, Chicago, The University of Chicago Press.

Clark, X., D. Dollar y A. Micco (2004), "Port efficiency, maritime transport costs, and bilateral trade", Journal of Development Economics, vol. 75, $\mathrm{N}^{\circ} 2$, Amsterdam, Elsevier.

COCHILco (Comisión Chilena del Cobre) (2007), "El mercado del ácido sulfúrico en Chile proyectado al año 2015" [en línea] http:// www.cochilco.cl/anm/articlefiles/614-INFORMEASULF2006_ VersionFINAL.pdf.

Cox, D. y W. Smith (1961), Queues, Nueva York, John Wiley.

De Graba, P. (1995), "Buying frenzies and seller-induced excess demand", The RAND Journal of Economics, vol. 26, $\mathrm{N}^{\circ} 2$, Wiley.

Engel, E., R. Fischer y A. Galetovic (2004), "How to auction a bottleneck monopoly when underhand vertical agreements are possible", Journal of Industrial Economics, vol. 52, $\mathrm{N}^{\circ} 3$, Blackwell Publishing.

Ghemawat, P. (1986), "Sustainable advantage", Harvard Business Review, Boston, Harvard Business Publishing, septiembre.

Ghosh, M. (2002), "Bidding for a berth: an action based queue management mechanism for ports", Singapore Maritime \& Port Journal, Singapur, Maritime \& Port Authority of Singapore.

Greenberger, M. (1966), "The priority problem and computer time sharing", Management Science, vol. 12, $\mathrm{N}^{\circ} 11$, Institute for Operations Research and the Management Sciences.

Haddock, D. y F. McChesney (1994), "Why do firms contrive shortages? The economics of intentional mispricing", Economic Inquiry, vol. 32, $\mathrm{N}^{\circ} 4$, Wiley.

Imai, A., K. Nagaiwa y W. Chan (1997), "Efficient planning of berth allocation for container terminals in Asia", Journal of Advanced Transportation, vol. 31, $\mathrm{N}^{\circ} 1$, Wiley.

Imai, A., E. Nishimura y S. Papadimitriou (2004), "Berth allocation with service priority", Transportation Research Part B: Methodological, vol. 37, $\mathrm{N}^{\circ}$ 5, Amsterdam, Elsevier.

Jansson, J. e I. Ryden (1979), Swedish Seaports: Economics and Policy, Estocolmo, Stockholm School of Economics, The Economic Research Institute.
Likens, J. (1976), "The welfare cost of nonoptimal airport utilization", Journal of Public Economics, vol. 5, $\mathrm{N}^{\circ} 1-2$, Amsterdam, Elsevier.

MacKinnon, J. y N. Olewiler (1980), "Disequilibrium estimation of the demand for copper", Bell Journal of Economics, vol. 11, $N^{\circ} 1$, Santa Monica, RAND Corporation.

Mancero, X. y E. Saavedra (2006), "Un modelo de entrada y competencia en telecomunicaciones", Revista de Análisis Económico, vol. 21, $\mathrm{N}^{\circ}$ 1, ILADES/Georgetown University.

Mandy, D. y D. Sappington (2007), "Incentives for sabotage in vertically-related industries", Journal of Regulatory Economics, vol. 31, $\mathrm{N}^{\circ} 3$, Springer.

Naor, P. (1969), "The regulation of queue size by levying tolls", Econometrica, vol. 37, $\mathrm{N}^{\circ} 1$, Nueva York, The Econometric Society.

Pestalozzi, G. (1964), "Priority rules for runway", Operations Research, vol. 12, $\mathrm{N}^{\circ} 6$, Institute for Operations Research and the Management Sciences.

Robinson, J. y L. Casalino (1996), "Vertical integration and organizational networks in healthcare", Health Affairs, vol. 15 , $\mathrm{N}^{\circ}$ 1, Bethesda, Project HOPE - The People-to-People Health Foundation, Inc. .

Sherman, R. y M. Visscher (1982), "Non-price rationing and monopoly price structures when demand is stochastic", The Bell Journal of Economics, vol. 13, $\mathrm{N}^{\circ} 1$, Santa Monica, RAND Corporation.

Slade, M. (1991), "Strategic pricing with customer rationing: the case of primary metals", Canadian Journal of Economics, vol. 24, $\mathrm{N}^{\circ} 1$, Wiley.

Strandenes, P. y E. Wolfstetter (2005), "Efficient (re-)scheduling: an auction approach", Economics Letters, vol. 89, $\mathrm{N}^{\circ} 2$, Amsterdam, Elsevier.

Svendsen, A.S. (1967), Does the Traditional Set-up of Port Charges Favour Old and Unmodern Ships?', Bergen, Institute of Shipping Research.

Ungem-Sternberg, T. (1991), "Rationing in restaurants", International Journal of Industrial Organization, vol. 9, $\mathrm{N}^{\circ}$ 2, Amsterdam, Elsevier.

Valetti, T. y C. Cambini (2005), "Investments and network competition", RAND Journal of Economics, vol. 36, N² ${ }^{\circ}$, Santa Monica, RAND Corporation. 